\title{
TU/e EN⿴HONE

\section{Performance evaluation of an optical transparent access tier based on PON and spectral codes}

\section{Citation for published version (APA):}

Huiszoon, B., Hernandez, J. A., Waardt, de, H., Khoe, G. D., Aracil, J., \& Koonen, A. M. J. (2009). Performance evaluation of an optical transparent access tier based on PON and spectral codes. IEEE Journal on Selected Areas in Communications, 27(2), 143-155. https://doi.org/10.1109/JSAC.2009.090206

DOI:

10.1109/JSAC.2009.090206

Document status and date:

Published: 01/01/2009

\section{Document Version:}

Publisher's PDF, also known as Version of Record (includes final page, issue and volume numbers)

\section{Please check the document version of this publication:}

- A submitted manuscript is the version of the article upon submission and before peer-review. There can be important differences between the submitted version and the official published version of record. People interested in the research are advised to contact the author for the final version of the publication, or visit the $\mathrm{DOI}$ to the publisher's website.

- The final author version and the galley proof are versions of the publication after peer review.

- The final published version features the final layout of the paper including the volume, issue and page numbers.

Link to publication

\section{General rights}

Copyright and moral rights for the publications made accessible in the public portal are retained by the authors and/or other copyright owners and it is a condition of accessing publications that users recognise and abide by the legal requirements associated with these rights.

- Users may download and print one copy of any publication from the public portal for the purpose of private study or research.

- You may not further distribute the material or use it for any profit-making activity or commercial gain

- You may freely distribute the URL identifying the publication in the public portal.

If the publication is distributed under the terms of Article 25fa of the Dutch Copyright Act, indicated by the "Taverne" license above, please follow below link for the End User Agreement:

www.tue.nl/taverne

Take down policy

If you believe that this document breaches copyright please contact us at:

openaccess@tue.nl

providing details and we will investigate your claim. 


\title{
Performance Evaluation of an Optical Transparent Access Tier Based on PON and Spectral Codes
}

\author{
B. Huiszoon Member, IEEE, J. A. Hernández, H. de Waardt Associate Member, IEEE, G. D. Khoe Fellow, IEEE, \\ J. Aracil Senior Member, IEEE and A.M.J. Koonen Fellow, IEEE
}

\begin{abstract}
The increasing amount of bandwidth requirements and quality of service needs for the next-generation access networks has boosted extensive research in the fiber-optics communication field. In this light, passive optical networks (PONs) combined with optical code division multiple access (OCDMA), provide a potentially cost-effective solution to meet such bandwidth demands. This work proposes an optical transparent architecture which enables all-optical communication between the network nodes. The encoded data streams are multiplexed at a merging point which results in multiple user interference (MUI), thus significantly reducing the network throughput. The networking nodes are able to monitor and record user activity in the PON, and further register the (past) state of activity at the merging point. In this work, we study the coherence of state between the networking nodes and the merging point, for different packet size distributions, in order to predict an optimal transmission instant of each node's data packets. We note that the states are coherent depending on the packet size distribution.
\end{abstract}

Index Terms-Code division multiaccess; Coherence of state; Network performance; Optical fiber communication; Passive optical networks.

\section{INTRODUCTION}

A $\mathrm{T}$ THE TIME of writing, it is estimated that over one billion $\left(10^{9}\right)$ people worldwide have access to the Internet [1]. A large-scale measurement experiment recently disclosed important details of commercial access networks in the USA and Europe [2]. Essentially, the traditional digital subscriber line (DSL) and coaxial cable (COAX) networks were evaluated in terms of up/downstream bandwidth (BW), packet latency and packet loss. The measured link BWs were much lower than future residential BW requirements, which have been estimated in the range from $2 / 20$ Mbps [3] to 30/100 Mbps [4]. Additionally, although measured bandwidths have been observed highly asymmetrical, the authors in [5] have shown that traffic patterns are becoming more and more symmetrical. Also the values measured for packet jitter and

Manuscript submitted 15 January 2008; revised 05 August 2008. This work has been a joint research within the FP6 e-Photon/ONe+ and the FP7 BONE Networks of Excellence. Acknowledged for funding are the Netherlands Organisation for Scientific Research (NWO), the Spanish Ministry of Science and Education and the European Commission.

B. Huiszoon, H. de Waardt, G.D. Khoe and A.M.J. Koonen are with the COBRA Institute of the Eindhoven University of Technology (TU/e), Den Dolech 2, PO BOX 513, 5600MB Eindhoven, Netherlands (e-mail: b.huiszoon@ieee.org).

J.A. Hernández and J. Aracil are with the Networking Research Group of the Departamento de Ingeniería Informática, Universidad Autónoma de Madrid, Calle Tomás y Valiente, 28049 Madrid, Spain (e-mail: jose.hernandez@uam.es).

Digital Object Identifier 10.1109/JSAC.2009.090206. queueing delay differ significantly from recommended values for interactive services [6]. Therefore, a low quality of service (QoS) is to be expected in DSL and COAX networks for future bandwidth-intensive and time-critical services. Moreover, the network infrastructure has to cope with the increasingly popular peer-to-peer networking which is becoming the dominant application in terms of transmitted traffic volumes. Considering the above, only a fiber-optic network is able to provide a future-proof solution.

This work proposes an optical transparent access tier to meet the requirements for the next-generation access networks. The architecture is based on passive optical networks and optical codes for shared medium access. Recently, promising experimental results have been shown using different optical coding techniques [7], [8]. The encoded data streams of networking nodes are asynchronously combined into a single stream at a merging point and may be passively broadcasted even to nodes located in other PONs connected. This way alloptical node-to-node communication is permitted which is an important added value, especially in access networks.

Inherently, the use of optical codes implies soft capacity degradation, which decreases the effective network throughput if not properly managed. In this work, the optical nodes have the ability to sense the signal level at the merging point, and even infer the user activity. This can be exploited in scheduling network access, as previously reported in [9] employing incoherent time-domain optical codes.

This paper analyzes the coherence of state between the optical node and the merging point where the data streams are multiplexed. That is, it is studied whether or not the level of activity sensed by the optical node remains stable with respect to the activity at the merging point. Unlike the authors in [9], this work proposes the use of orthogonal codes that do not employ time-domain chips and we further analyze the coherence of state from a packet level. The analysis also assumes different packet size distributions, and a bimodal distribution that accurately captures the measured packet size distribution in real networks [10]. Finally, coherence of state has a direct application in the definition of medium access control (MAC) protocols that control the access to the passive optical network. By sensing the activity at the merging point, the end optical nodes have a means to decide the appropriate transmission instant of its packets, on attempts to reduce interference.

The remainder of this work is organized as follows: Section II motivates the use of an optical transparent tier at 
the network access, showing its benefits in three emerging network scenarios. Then, the underlying physical architecture is presented in section III and it is shown that its performance is mainly limited by the interference between active users. A key functionality, all-optical traffic monitoring, is introduced at the end of section III which may be used to anticipate on the network activity. The coherence of state between the optical node and the merging point is further analyzed for different packet size distributions and user scenarios in section IV through section VI. Transmission scheduling is presented as a straightforward application of coherence of state in section VII. Finally, a summary of the main contributions of this work is given in section VIII.

In conclusion, this paper provides a novel architecture proposal in the network access, it further shows its limitation in terms of MUI, studies the validity of the monitored interference information via the coherence of state analysis, and proposes how to overcome this limitation via transmission scheduling. To the best of our knowledge, there is no other work that provides such a complete treatment of such optical transparent architecture.

\section{Motivation}

In this section, the main characteristics of fiber-optics are presented and optical transparency is shown to have added value. Three emerging network scenarios are discussed, namely personal, residential and business, which all benefit from an optical transparent access tier.

Fiber optics provide a strong alternative to alleviate the access bottleneck because of its extremely large bandwidth capacity, low propagation loss and signal transparency. Moreover, optical signal processing and switching speed exceed the capabilities in the electrical domain with less required power consumption per bit [11]. Optical access networks are increasingly being deployed either to replace the existing infrastructure because of lower operational expenses (OPEX), or in green-field situations because of lower capital expenses (CAPEX) [12]. Today's preferred optical architecture is the PON where multiple optical networking units (ONUs) are connected to a central office (CO) via a single passively split optical fiber. Such a topology becomes cost-efficient when the distance increases between the $\mathrm{CO}$ and ONUs and/or when the splitting ratio increases [13].

Downstream traffic is broadcasted from the $\mathrm{CO}$ to the ONUs while medium access has to be provided in case of upstream traffic. Conventional systems enable shared access via established time or wavelength division multiple access (TDMA, WDMA). Instead, optical codes are proposed (hence denoted OCDMA) which rely on communication via a unique and orthogonal code. The orthogonality of the code allows the carrier to be asynchronously shared with other users on the network. The primary application domain of OCDMA is the access network because it offers cost-effective network deployment and management combined with physical layer security [14]. Additionally, OCDMA has a natural fit on PON because both are based on broadcast-and-select. Moreover, in a multiple wavelength channel system it allows a code reuse per channel. Thus OCDMA offers cost-effective network access to a large number of subscribers on the reduced infrastructure costs of a PON.

Ubiquitous broadband connectivity and mobility are two network experiences which have become a commodity. Mobile users increasingly interconnect their personal devices to form their own short-range personal area network (PAN) which is enabled by Bluetooth or IEEE 802.15 [15]. The future communication needs of these subscribers is reflected in the recently introduced personal network (PN) concept for global connectivity to personalized networks [16]. If the PAN (a truly mobile network) is taken as an entity, a PN is defined by the PAN and the other remote personal devices the PAN is connected with. An optical transparent access network mitigates many issues currently encountered regarding connectivity and mobility management in PNs [17].

Recently, researchers have analyzed residential networks by studying its parameters, traffic characteristics [18] and implications on the higher network tiers. A conclusion shared by all studies is that peer-to-peer (P2P) communication remains to be a heavy load on the network. Small-world properties are attributed to typical P2P applications such as eMule and BitTorrent [19]. However, in practice, poor locality was shown because more than $70 \%$ of existing local content was downloaded from peers outside the domain of the internet service provider (ISP) [5], [20]. Locality-aware protocols have been proposed in order to reduce cross-ISP/cross-tier traffic [21]. Certainly, an increase of local traffic intensity is not supported by the access tier in its current state.

Reliable connections while accessing a shared network such as the Internet is an essential asset in a business environment [22]. Virtual private networks (VPNs) are mostly used because they offer cost savings and communication privacy, and can be implemented in different network layers [23]. Small- to medium-size enterprizes may lease a VPN for a costefficient business network deployment and operation. Large enterprizes or research institutes with a privately owned infrastructure may run secure connections if different (corporate) parties are using the network. A VPN implemented in Layer 1, or optical VPN (OVPN), is considered to be a key service in the next-generation transport network given its high speed, efficiency and low cost [24]. An optical transparent access layer has an obvious key role in establishing OVPNs.

In summary, the three network scenarios (personal, residential and business) and the particular benefits of transparent optical networks (reliability, reduced complexity, power efficiency and processing speeds) strongly motivate the introduction of optical technologies in the access domain. In the remaining of this work, a solution based on OCDMA and PONs is proposed and analyzed in detail as a potentially costeffective architecture to be deployed in an optical transparent access tier.

\section{PROPOSED NETWORK DESIGN}

In this section we discuss in detail the optical access network architecture and its parameters, the transmission techniques and traffic handling. Transparent user-to-user communication is proposed by employing optical codes. Out of the many available techniques, incoherent spectral amplitude 
coding is depicted as a candidate to implement such transmission scenario because of specific cost-reducing system characteristics. This section concludes with a brief discussion on code-based all-optical contention resolution schemes.

\section{A. Architecture}

The optical access network architecture is based on multiple passive optical networks connected to a single central office as depicted in Fig. 1 which is similar to [1]. As shown, the central office has $M$ PONs connected to it. Each of them has $N_{i}$ ONUs which is equal to the splitting ratio $N_{i}$ of the passive coupler. Obviously, the value $M$ is largely determined by the backplane of the CO while $N_{i}$ has a physical limitation via the power budget. The power budget determines the allowable losses on the path between a source and destination for a given transmitter power and receiver sensitivity. Current PON deployments use $N_{i}=32$ or $N_{i}=64$ as maximum splitting ratio which gives an ideal power loss of $10 \log \left(N_{i}\right)$ dB plus excess losses. Considering the fiber lengths we assume an urban area scenario meaning a distribution fiber of $1 \mathrm{~km}$ and a feeder fiber of $4 \mathrm{~km}$. As shown in Fig. 1, separated fibers are used for up and downstream transmission. The operating bit rate is the synchronous transfer mode (STM)-1 base rate (155.52 Mbps) in order to meet the future BW requirements.

\section{B. Transparent communication via optical codes}

The situation may occur when two users located at different ONUs are communicating with each other via the $\mathrm{CO}$ and, normally, such ONU-to-ONU (O2O) communication requires a conversion to the electronic domain in order to perform the routing of the data. All-optical communication between ONUs results in two additional $\mathrm{O} 2 \mathrm{O}$ traffic streams depending on the location of the destination. Both are shown in Fig. 1 next to the regular $\mathrm{CO}-\mathrm{ONU}$ traffic. All-optical $\mathrm{O} 2 \mathrm{O}$ communication has been studied in case of a single PON, i.e. [25], or recently, in case of a sub-net of PONs [26], [27]. The authors in [26] use time slots in a wavelength division multiplexing (WDM) scheme which require a central clock for synchronous access and accurate traffic scheduling to avoid contention. A synchronous time-slotted PON is a complex solution considering the application area. We proposed to use optical codes (OCs) instead of time slots on WDM PON and to enable O2O communication in a sub-net of PONs [27].

The $\mathrm{O} 2 \mathrm{O}$ data is transmitted on the optical address of the destination. The address is unique and composed by an optical wavelength and a code. Each individual bit is coded with the address instead of only an OC label in front of the data packet such as in optical code generalized multiprotocol label switching (OC-GMPLS) systems. In this work no wavelength conversion nor code conversion is applied in any node between two ONUs in order to have end-to-end truly all-optical communication.

\section{Address and traffic handling}

If data is ready for transmission, the ONU acquires the optical address of the destination at the $\mathrm{CO}$. We assume that a dedicated collision-free communication channel exists between the $\mathrm{CO}$ and ONUs which is used for transmission control and coordination according to a standard common control-channel architecture. The channel is placed out-ofband to not interfere with the data transmission channels. Three forms of address usage are possible namely fixed, semi-dynamic (e.g. the destination either has a fixed code or wavelength and tunes to an available wavelength or code) or dynamic (e.g. the destination may tune to any available address). Thus the transmitter is always tunable but a negligible tuning time is considered. The dynamic entity in the optical address may be assigned via, for example, a list-scheduling algorithm or a hash function.

Users A-D in Fig. 1 may be either fixed and/or mobile network subscribers depending on which network scenario is considered (see Section II). The data for fixed users located at $\mathrm{ONU}_{a, b}$ could be aggregated in a burst. If a mobile user moves from $\mathrm{ONU}_{a, b}$ to $\mathrm{ONU}_{a+1, b}$, the connection should remain with data routed accordingly. The wireless network service can be transparently deployed by using radio-over-fiber techniques as shown in [17]. The CO has a regularly updated list of all active users in the network. As such it keeps track at which ONU a user is located and registers movements from $\mathrm{ONU}_{a, b}$ to $\mathrm{ONU}_{a+1, b}$.

\section{Spectral amplitude optical coding}

A classification of the available optical coding techniques can be done via the correlation principle (coherent or incoherent), coding domain (time, frequency, or time and frequency), en/decoder implementation (free-space, fiber-based or integrated), and line code (unipolar or polar signaling). We consider the cost-effective spectral amplitude optical coding technique to further reduce the CAPEX and OPEX of the code-based optical access network [28].

Spectral amplitude optical coding employs incoherent correlation, frequency codes, integrated en/decoders (E/Ds) and polar signaling. Here the E/Ds are amplitude filters which imprint their filter pattern, $A(\omega)$, in the optical field of a broad spectral incoherent source to form a spectral code. This source may be a superluminescent light emitting diode (SLED). The E/D is based on the well-known unbalanced Mach-Zehnder interferometer (MZI) which naturally generates two complementary (or $\pi$-shifted) and periodic fringe patterns. The periodicity is defined as the free spectral range (FSR) of the device. Multi-staged configurations with tunable phase shifters are used to generate different, orthogonal filter patterns which represent the spectral codes.

The spectral code and its complementary, $\bar{A}(\omega)$, are used to transmit the binary data in the optical domain. For example, $A(\omega)$ is transmitted to represent the logical one and $\bar{A}(\omega)$ is sent to represent the logical zero. This type of modulation is denoted spectral shift keying (SSK). A similar tunable E/D, $B(\omega)$, is placed at the receiver side which is combined with a balanced detector. If $A(\omega)=B(\omega)$ the optical power at the $\mathrm{E} / \mathrm{D}$ output varies between a maximum and minimum value whereas if $A(\omega) \neq B(\omega)$ average optical power is received at both outputs. SSK combined with balanced detection leads to polar signaling and has a 3-dB signal to noise (SNR) ratio compared with on-off keying (OOK). Note that coding in 


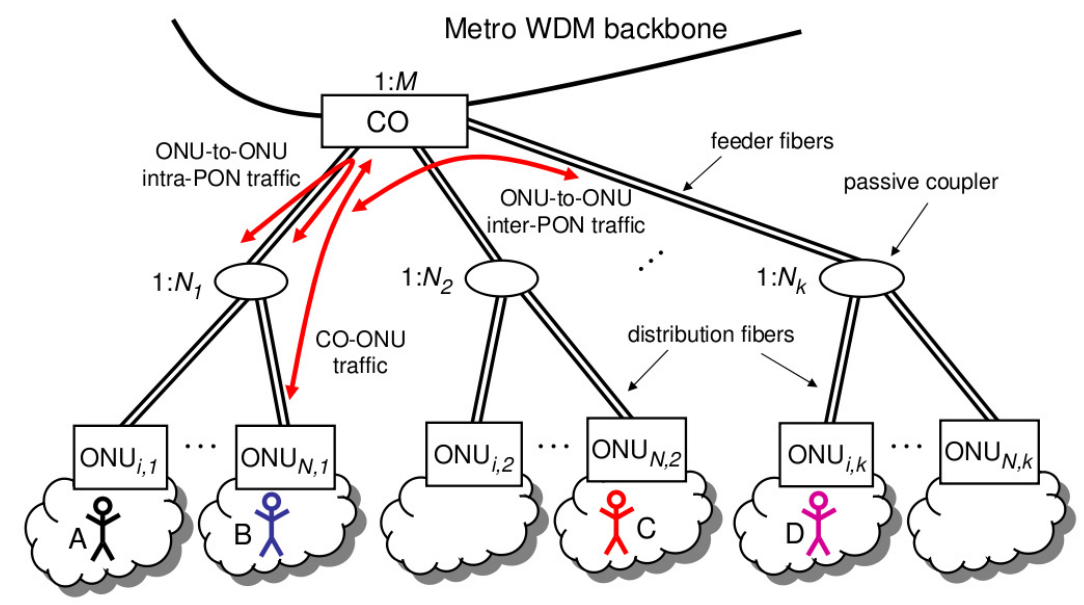

Fig. 1. Passive optical network-based access network and the traffic streams

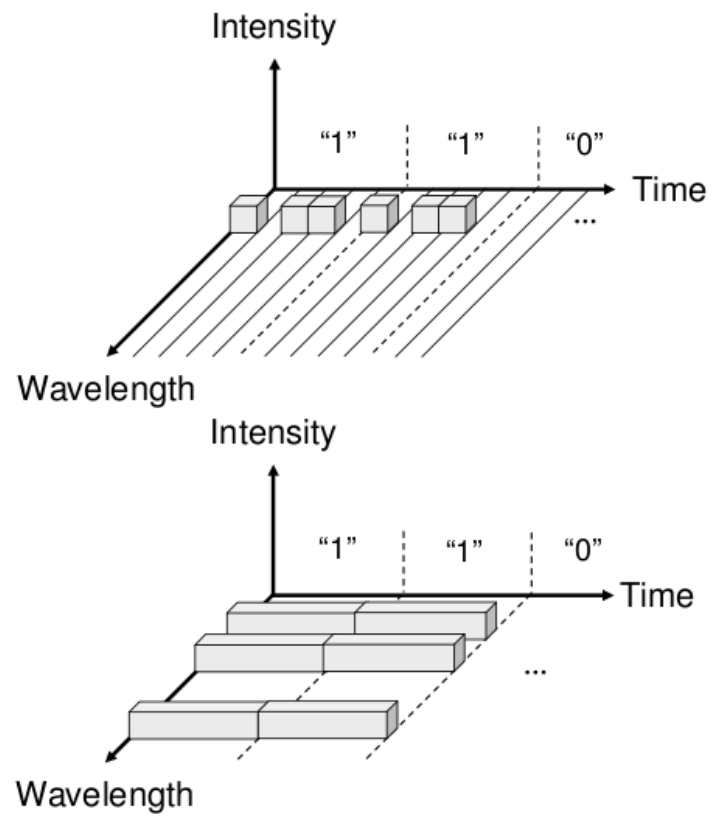

Fig. 2. Incoherent time vs spectral amplitude optical coding (three bits shown)

an SSK system is done at the bit rate $B$, rather than using chips at the chip rate $C$ of, for example, an incoherent timedomain OCDMA system which typically results in a lower required system bandwidth and optical sources with a lower complexity. The difference between an incoherent time and wavelength-domain OCDMA system is schematically shown in Fig. 2 for the bit sequence 110 .

A spectral amplitude encoded optical code multiple access (SAE OCDMA) PON is shown is shown in Fig. 3 for an upstream configuration. As shown, the output of the SLED is filtered by the bandpass filter (BPF) which $-3 \mathrm{~dB}$ bandwidth equals to one period (or FSR) of the MZ-based cascade E/D. The spectral slice is led into the optical switch to perform the SSK modulation by alternatively launching the optical power at the upper or the lower input of the encoder. The ONU is able to tune to an available wavelength(-band) in a
WDM scheme. An optical amplifier finally compensates for the losses experienced by the coding and switch. The encoded optical stream is launched onto the distribution fiber $L_{d}$ and multiplexed with other streams at the passive coupler. At the $\mathrm{CO}$ in Fig. 3, the optical stream is received by a tree E/D which is a parallel code processing element, and balanced detection. The system nodes shown in Fig. 3 are fully integrateable into an optical chip with other optical functions, which enables large-scale system deployment by means of mass production.

\section{E. Contention resolution and activity monitoring}

Collisions are at stake when multiple ONUs in Fig. 1 want to send data upstream to the same destination. All-optical code-labeled contention resolution has been proposed and analyzed in [29] for such a network. A combination of optical code-sense medium access /collision detection (OCSMA/CD) and code-labeled packet switching has been proposed. The feasibility of implementing such methods has to be carefully considered therefore a number of issues such as the scalability and complexity is addressed.

OCSMA/CD requires a reflective PON, i.e. the optical power of a transmitting ONU is reflected to all other ONUs except to itself. Let us assume that a given ONU is equipped with code sensing capabilities such that it can detect how many codes or users are active. A proposed design is depicted in Fig. 4 which is able to perform OCSMA/CD combined with code activity monitoring for a specific optical channel. As mentioned before, the ONU receives the transmitted data of the other ONUs which are directed to the tree E/D via the optical circulator after selecting the transmission band with the tunable BPF. The transmission scheduling module also contains a means to store data in an electronic buffer. Recently, a similar tree E/D has been used in field experiments employing coherent wavelength-domain OCs [8] which indicates its potential. The design in Fig. 4 can be fully integrated using hybrid techniques on Silicon.

\section{Performance evaluation}

A well-known limiting factor of OCDMA networks is the multiple user (or access) interference. In this section, we 


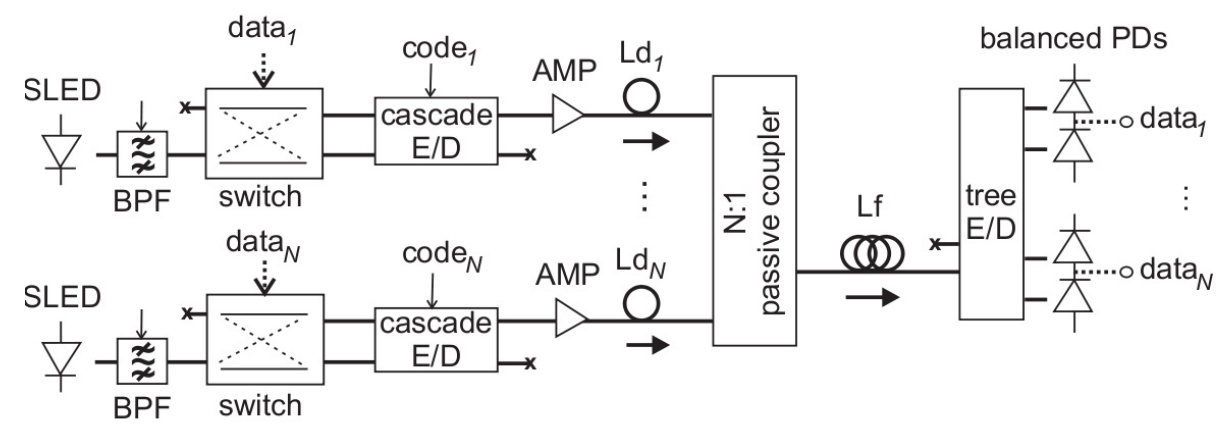

Fig. 3. Basic SAE-OCDMA/WDM PON, upstream configuration

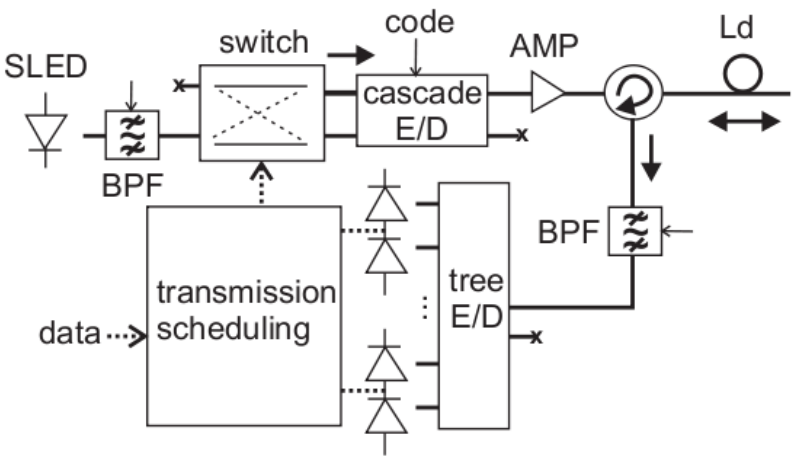

Fig. 4. Code activity monitoring functionality in SAE-OCDMA ONU

provide a performance evaluation in terms of the bit error rate (BER) caused by mutual interference.

\section{A. Multiple user interference}

As more users simultaneously access the medium, the interference between codes increases at the expense of network throughput. For example, consider an incoherent time-encoded OCDMA system. At the transmitter-side, fiber delay lines apply time-shifts to arrange pulses in order to create an orthogonal sequence. At the receiver, the opposite configuration is used to reconstruct the original high-intensity data signal via incoherent summation of the pulses. Random network access by multiple users may lead to the so-called false positives at receivers (i.e. bit detection where no bit was actually transmitted), which is caused by the summation of time-shifted codes.

A promising method to reduce the MUI in this system was proposed in [30] and experimentally confirmed in [31]. In their approach, interference avoidance (IA) decides the ideal bit release time with respect to the transmitted signals sensed on the line in order to avoid interference, thereby improving throughput. The IA protocol operates at the chip (rate) level. In the case of SAE OCDMA, interference is experienced as long as different codes overlap. Moreover SSK also transmits a code to represent a logical zero while in [30] OOK is used. As such, the interference in the SAE OCDMA system occurs asynchronously during the whole duration of the packet. Under the assumption of Internet protocol (IP) packet transmission, a single bit error directly translates into packet drop at the next IP hop, regardless of the number of bits involved.

A fundamental assessment of the MUI in SSK SAEOCDMA is shown in [32] where the noise power at the balanced detector is evaluated. The noise can be modeled by the sum of a number of individual noise components, namely thermal, shot and speckle noise. The latter refers to the intensity fluctuations caused by the incoherent summation of signal powers when spectral codes overlap. This noise source is denoted speckle noise by [32] because it is a similar mechanism that gives rise to the spatial intensity variations in coherent images called "speckle". In order to avoid confusion, this work refers to the noise source as incoherent speckle noise. Incoherent speckle noise is a major contributor to MUI and is even present in the absence of interfering users. The components of the total noise power are given as follows (the derivation of the equations can be found in [32]). Note that modulator noise, RF amplifier noise and other noise sources are not included in the analysis.

The thermal noise $\left\langle I_{\mathrm{th}}^{2}\right\rangle$ is given by

$$
\left\langle I_{\mathrm{th}}^{2}\right\rangle=8 \pi k T B_{\mathrm{d}}^{2} C
$$

with $k$ the Boltzmann constant, $T$ the temperature in Kelvin, $B_{\mathrm{d}}$ the detector bandwidth in Hertz and $C$ the load capacitance in Farad. The shot noise $\left\langle I_{\mathrm{sh}}^{2}\right\rangle$ follows

$$
\left\langle I_{\mathrm{sh}}^{2}\right\rangle=2 q \Re P_{\text {rec }} u B_{d}
$$

with $q$ the charge of an electron in Coulomb, $\Re$ the responsivity of the photodetector, $P_{\text {rec }}$ the received power in Watt, and $u$ the number of active users. Finally the incoherent speckle noise $\left\langle I_{\mathrm{sp}}^{2}\right\rangle$ is described by

$$
\left\langle I_{\text {sp }}^{2}\right\rangle=\Re^{2} P_{\text {rec }}^{2}\left(u^{2}+1\right) \frac{2 B_{\mathrm{d}}}{m M B_{\text {opt }}}
$$

with $m=1$ for polarized light and $m=2$ for unpolarized light, $M$ the number of modes in the fiber and $B_{\text {opt }}$ the spectral width of the code in Hertz. The total noise contribution $\left\langle I_{n}^{2}\right\rangle$ at the detector is then

$$
\left\langle I_{\mathrm{n}}^{2}\right\rangle=\left\langle I_{\mathrm{th}}^{2}\right\rangle+\left\langle I_{\mathrm{sh}}^{2}\right\rangle+\left\langle I_{\mathrm{sp}}^{2}\right\rangle .
$$

On the other hand, the signal power is given by $I_{\text {sig }}^{2}=\Re^{2} P_{\text {rec }}^{2}$. Thus, given a number of users $u$, the bit error rate $\left(\mathrm{BER}_{u}\right)$ 


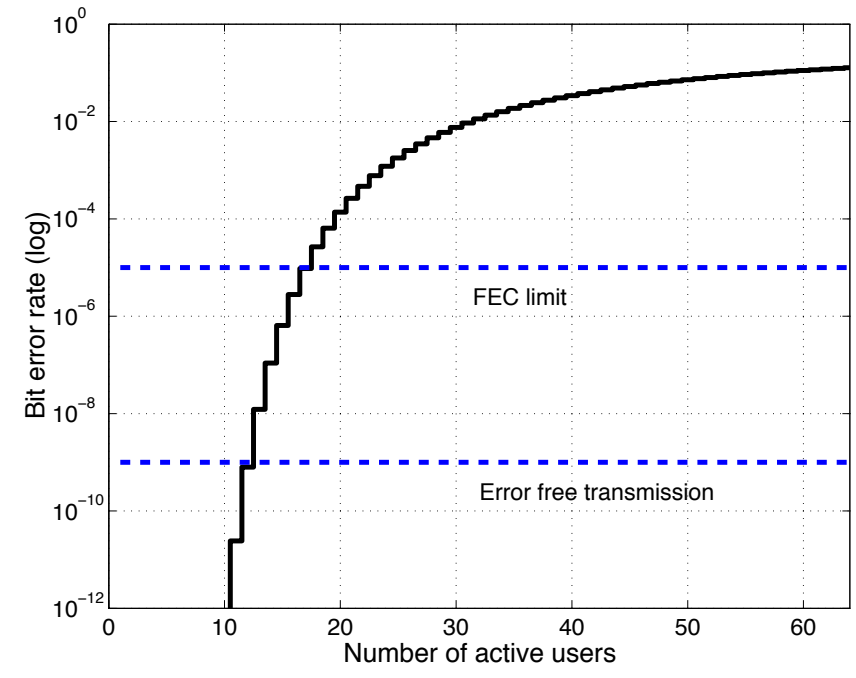

Fig. 5. BER versus active users in SAE OCDMA system

that they experience is calculated as [32]

$$
B E R_{u}=\frac{1}{2}\left[1-\operatorname{erf}\left(\sqrt{\frac{I_{\text {sig }}^{2}}{\left\langle I_{\mathrm{n}}^{2}\right\rangle}}\right)\right]
$$

The following realistic parameters apply to our system, namely: $\Re=0.71, P_{\text {rec }}=10^{-6} \mathrm{~W}$ (per user), $T=293 \mathrm{~K}$, $C=0.02 \cdot 10^{-12} \mathrm{C}, m=M=1, B_{\mathrm{d}}=0.75 \cdot B \mathrm{~Hz}$, $B_{\text {opt }}=624 \cdot 10^{9} \mathrm{~Hz}(=5 \mathrm{~nm}$ at $1550 \mathrm{~nm})$. With these values, Fig. 5 shows the calculated $\mathrm{BER}_{u}$ versus $u$ (the number of active users) assuming $B=155.52 \mathrm{Mbps}$. The detrimental effect of MUI in an SAE OCDMA system becomes clear, since the more users transmitting simultaneously, the higher the bit error rate experienced. In the figure, the error-free $\left(\mathrm{BER}=10^{-9}\right)$ limit and a forward error correction (FEC) limit of $10^{-5}$ are exceeded when more than twelve and seventeen users are active, respectively. Authors in [33] show that a BER of $3.3 \cdot 10^{-3}$ may be corrected to $10^{-9}$ for a $7 \%$ FEC overhead. Therefore, the limit of $10^{-5}$ introduces a margin in order to alleviate strict requirements to implement FEC in the noisy SAE-OCDMA environment. As indicated by [32], an increase in the received power only improves performance when multimode fiber (MMF, $M>1$ ) is used. Other solutions to reduce BER comprise either the increase of $B_{\mathrm{opt}}$ on attempts to spread the effect of incoherent speckle noise or a reduction of the bit rate. Additionally, Fig. 5 shows that the SAE OCDMA system performance does not depend on which particular codes are being used on the network. This characteristic, combined with the fact that interference is experienced at packet level, deduces the objective to minimize the code activity on the network based on the interference sensed on the line.

\section{B. Average BER versus network load}

Let $R_{u}$ be the average packet or burst arrival rate per user and let $1 / \tau_{\text {av }}$ be the average transmission time. Then, $\rho=R_{u} / \tau_{\text {av }}$ is the utilization factor of the output queue at the ONU, smaller than one for stable queues. Note that, from direct application of the well-known PASTA (Poisson Arrivals See Time Averages) property, the utilization factor is

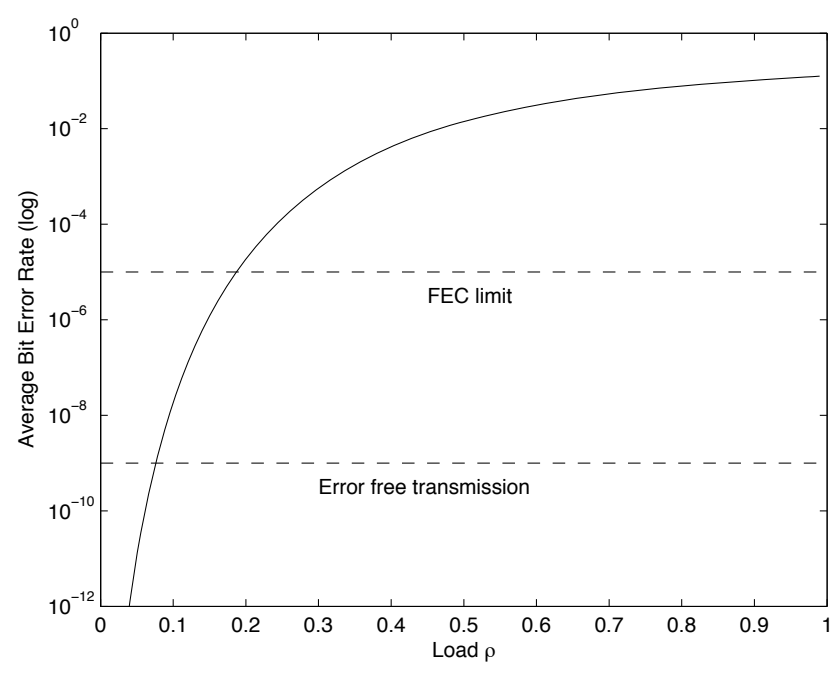

Fig. 6. Average BER for different load levels $\rho$

the probability to find the queue is busy, as seen by a random (Poisson) arrival. Furthermore, this result holds regardless of the packet or burst size distribution. Then, it can be assumed that a given user is active (i.e. transmitting a data packet or burst) with probability $\rho$. The probability $p(u)$ to find exactly $u$ active users out of a total population of $U$ users is then given by the following Binomial distribution

$$
p(u)=\left(\begin{array}{l}
U \\
u
\end{array}\right) \rho^{u}(1-\rho)^{U-u}, \quad u=0, \ldots, U
$$

with $U=64$ ONUs, as noted in section III.

Eq. 6 above can be combined with Eq. 5 to obtain the average BER probability (denoted $\overline{B E R}$ ) as a function of the number of users, as given by

$$
\overline{B E R}=\sum_{u=0}^{U} B E R_{u} p(u)
$$

Eq. 7 has been plotted in Fig. 6 for several values of $\rho$.

For example, the chosen FEC limit of $10^{-5}$ imposes a load operation below $20 \%$ in this architecture, as shown in Fig. 6 .

\section{COHEREnCE OF STATE ANALYSis}

Coherence of state is defined as a property by which state at a measurement point and instant is correlated to the state in other measurement point and instant. In [9], the state of the line at the $\mathrm{ONU}$ and the merging point is found coherent via the Pearson's correlation coefficient, under the assumption of exponential packet or burst size distributions.

In the forthcoming, a more rigorous estimation of such coherence of state is derived which takes into account other packet size distributions and also an empirically-observed bimodal distribution. The reader should note that such analysis is performed at the packet level, rather than at the chip level as pointed out in Section IV, given the particular characteristics of the optical codes used. The equations derived shall be evaluated with numerical examples in the next section.

The problem can be stated as follows: Given a set of observations of past "activity" by one of the ONUs, it is 


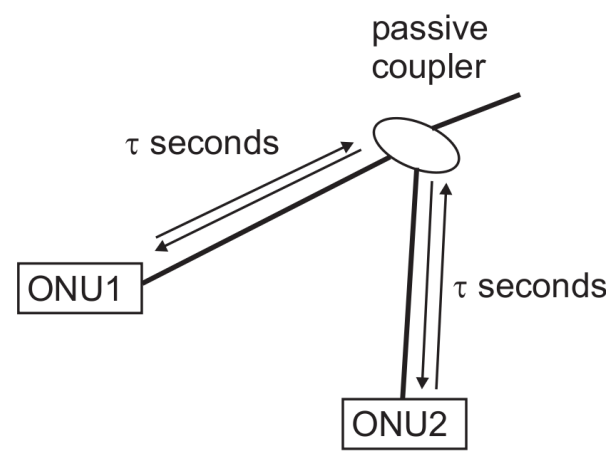

Fig. 7. Basic two-user OCDMA PON transmission scenario

desired to find the probability that such activity will remain at the merging point $d$ seconds into the future $(d \geq 0)$, as shown in Fig. 7. For the sake of simplicity, both ONUs in Fig. 7 are assumed equidistant with respect to the passive coupler i.e. fixed delay $\tau$ is assumed), and only the upstream direction is considered.

The following studies the case for single and multiple active users.

\section{A. Single active user}

Let $X$ be the packet duration. Let $\left\{Y_{1}, Y_{2}, \ldots, Y_{n}, \ldots\right\}$ be the sampling time instants, with $Y_{n}=n \cdot \delta$ and $\delta$ is the sampling interval. Let $Y_{n}=1$ if activity is sensed and zero otherwise. Our aim is to calculate

$P\left(X>n \delta+d \mid Y_{1}=1, \ldots, Y_{n}=1\right)=P(X>n \delta+d \mid X>n \delta)$

for $\delta>0$ and different packet length distributions, namely an exponential, Gaussian, Pareto and an empirically-observed bimodal distribution.

1) Exponential random variable: If the packet duration $X$ is exponentially distributed, with parameter $\mu$, then, due to its memoryless property it turns out that

$$
P\left(X>n \delta+d \mid Y_{1}=1, \ldots, Y_{n}=1\right)=P(X>d)=e^{-\mu d}
$$

2) Pareto random variable: If $X$ is Pareto of the second kind (Lomax) with tail index $\alpha$, that is

$$
P(X \geq x)=(x+1)^{-\alpha}, x \geq 0
$$

then

$$
\begin{aligned}
P(X>n \delta+d \mid X>n \delta) & =\frac{(1+n \delta+d)^{-\alpha}}{(1+n \delta)^{-\alpha}} \\
& =\left(\frac{1+n \delta}{1+n \delta+d}\right)^{\alpha}
\end{aligned}
$$

It is worth mentioning that for $1<\alpha \leq 2$ the Pareto distribution has an infinite variance with a finite mean which represents a heavy-tailed packet length distribution.

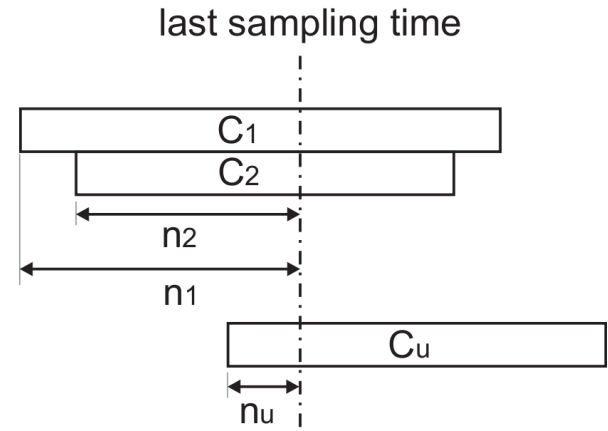

Fig. 8. Asynchronously multiplexed encoded data packets

3) Gaussian random variable: If $X$ is a Gaussian random variable with mean $\mu$ and standard deviation $\sigma$ then

$$
P(X>n \delta+d \mid X>n \delta)=\frac{1-\Phi\left(\frac{n \delta+d-\mu}{\sigma}\right)}{1-\Phi\left(\frac{n \delta-\mu}{\sigma}\right)}
$$

where $\Phi(\cdot)$ is the distribution function of the standard Gaussian random variable.

4) Bimodal random variable: It has been observed in previous measurement studies that the packet size distribution typically follows a bimodal distribution, that can be accurately characterized by the following combination of two Gaussian distributions (see [10]):

$$
P(X=x)=\alpha_{1} N\left(\mu_{1}, \sigma_{1}\right)+\alpha_{2} N\left(\mu_{2}, \sigma_{2}\right), \quad x \geq 0
$$

where $N(\mu, \sigma)$ is the probability density function (PDF) of a Gaussian distribution with mean $\mu$ and standard deviation $\sigma$. The values used in [10] to empirically characterize the packet size distribution are $\alpha_{1}=0.74, \mu_{1}=127$ bytes, $\alpha_{2}=0.26$, $\mu_{2}=1366$ bytes, and $\sigma_{1}=\sigma_{2}=20$ bytes. In this case:

$$
\frac{P(X>n \delta+d \mid X>n \delta)}{1-\left[\alpha_{1} \Phi\left(\frac{n \delta+d-\mu_{1}}{\sigma_{1}}\right)+\alpha_{2} \Phi\left(\frac{n \delta+d-\mu_{2}}{\sigma_{2}}\right)\right]}=
$$

\section{B. Multiple active users}

Consider multiple active users $u=1,2, \ldots, U$ in the network, each of them transmitting with its own code $C_{1}, C_{2}, \ldots, C_{u}$, and let $n_{1}, \ldots, n_{u}$ be the past activity intervals as sensed by a given ONU. Without loss of generality, let us assume that $n_{1} \geq n_{2} \geq \ldots \geq n_{u}$, as illustrated in Fig. 8 .

Coherence of state refers to the probability to have all $u$ users active $d$ seconds later, whereby a fixed propagation delay $\tau$ is assumed. Then, in order to have all users active $d$ seconds in the future, it is a necessary and sufficient condition that the minimum of them remain active for $d$ seconds in the future, i.e (15).

Clearly $\frac{P\left(X_{i}>n_{i} \delta+d\right)}{P\left(X_{i}>n_{i} \delta\right)}<1, i=1, \ldots, u$ which directly implies that $\lim _{u \rightarrow \infty} P\left(\min \left(X_{1}, \ldots, X_{u}\right)>n_{u} \delta+d \mid X_{1}>\right.$ $\left.n_{1} \delta, \ldots, X_{u}>n_{u} \delta\right)=0$. Namely, as the number of active 

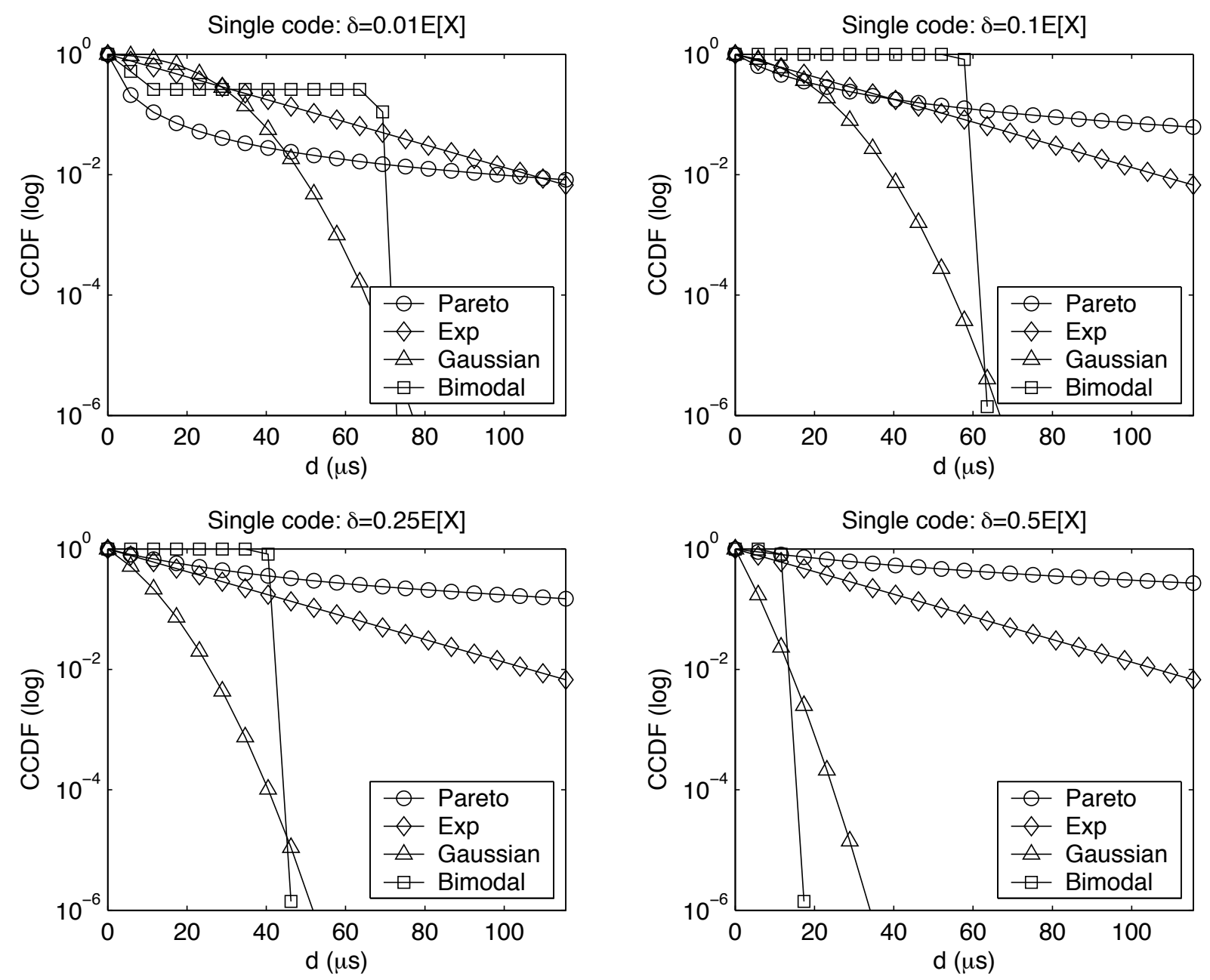

Fig. 9. Comparison sampling instants: $0.01 E X$ (top-left), $0.1 E X$ (top-right), $0.25 E X$ (bottom-left) and $0.5 E X$ (bottom-right).

$$
P\left(\min \left(X_{1}, \ldots, X_{u}\right)>n_{u} \delta+d \mid X_{1}>n_{1} \delta, \ldots, X_{u}>n_{u} \delta\right)=\prod_{i=1}^{u} \frac{P\left(X_{i}>n_{i} \delta+d\right)}{P\left(X_{i}>n_{i} \delta\right)}, \quad d \geq 0
$$

users increases the coherence of state approaches null. Furthermore, $P\left(\min \left(X_{1}, \ldots, X_{u}\right)>n_{u} \delta+d \mid X_{1}>n_{1} \delta, \ldots, X_{u}>\right.$ $\left.n_{u} \delta\right)$ is strictly decreasing with $u$, the number of active users. Also, the more active users sensed, the less coherence of state between the merging point and the optical node. Intuitively, when a large number of codes are present, it is more unlikely to have all of them active for $d$ seconds into the future.

The following solves Eq. 15 for different packet size distributions.

1) Exponential random variable: In this case, (16) where $1 / \mu_{i}$ is the mean packet duration for each code. If all packets are assumed to have the same average size, the above simply yields $P\left(\min \left(X_{1}, \ldots, X_{u}\right)>n \delta+d \mid X_{1}>n_{1} \delta, \ldots, X_{u}>\right.$ $\left.n_{u} \delta\right)=e^{-u \mu d}$.

2) Pareto random variable: In this case, (17).

3) Gaussian random variable: In this case, (18).

4) Bimodal random variable: In this case, (19).

\section{COHERENCE OF STATE EXPERIMENTS}

In this section, a number of numerical examples are proposed to evaluate the coherence of state between a given $\mathrm{ONU}$ and the passive coupler when either a single user is transmitting or a number of them.

\section{A. Simulation parameters}

In all packet size distributions, the mean value has been assumed $E X=0.74 \times 127+0.26 \times 1366=449.14$ bytes, since this is the average empirical packet size [10]. This gives an average transmission delay of $23.14 \mu \mathrm{s}$ at $B=155.52$ Mbps. For Pareto distributed packet sizes, the simulation value of $\alpha$ was $\alpha=1.2$, and for Gaussian packet lengths, $\sigma_{X}=0.5 E X$. Finally, the sampling instant was $5 \delta$, where $\delta \in\{0.01 E X, 0.1 E X, 0.25 E X, 0.5 E X\}$, and an optical fiber length $L=1 \mathrm{~km}$ which yields a propagation delay $\tau=4.8$ $\mu \mathrm{s}$. With these parameters, the following shows the coherence of state assuming a single active user, and multiple active 


$$
\begin{gathered}
P\left(\min \left(X_{1}, \ldots, X_{u}\right)>n_{u} \delta+d \mid X_{1}>n_{1} \delta, \ldots, X_{u}>n_{u} \delta\right)=e^{-\sum_{i=1}^{u} \mu_{i} d} \\
P\left(\min \left(X_{1}, \ldots, X_{u}\right)>n_{u} \delta+d \mid X_{1}>n_{1} \delta, \ldots, X_{u}>n_{u} \delta\right)=\prod_{i=1}^{u}\left(\frac{1+n_{i} \delta}{1+n_{i} \delta+d}\right)^{\alpha} \\
P\left(\min \left(X_{1}, \ldots, X_{u}\right)>n_{u} \delta+d \mid X_{1}>n_{1} \delta, \ldots, X_{u}>n_{u} \delta\right)=\prod_{i=1}^{u} \frac{1-\Phi\left(\frac{n_{i} \delta+d-\mu}{\sigma}\right)}{1-\Phi\left(\frac{n_{i} \delta-\mu}{\sigma}\right)} \\
P\left(\min \left(X_{1}, \ldots, X_{u}\right)>n_{u} \delta+d \mid X_{1}>n_{1} \delta, \ldots, X_{u}>n_{u} \delta\right)=\prod_{i=1}^{u} \frac{1-\left[\alpha_{1} \Phi\left(\frac{n_{i} \delta+d-\mu_{1}}{\sigma_{1}}\right)+\alpha_{2} \Phi\left(\frac{n_{i} \delta+d-\mu_{2}}{\sigma_{2}}\right)\right]}{1-\left[\alpha_{1} \Phi\left(\frac{n_{i} \delta-\mu_{1}}{\sigma_{1}}\right)+\alpha_{2} \Phi\left(\frac{n_{i} \delta-\mu_{2}}{\sigma_{2}}\right)\right]}
\end{gathered}
$$

users, for all packet size distributions discussed in the previous section.

\section{B. Single user experiments}

Fig. 9 shows the complementary cumulative distribution function (CCDF) for the four packet size distributions under study. As shown, exponential (memoryless) behavior shows a straight line CCDF, whereas the Gaussian and Pareto show faster-than and slower-than exponential decay respectively. The bimodal distribution appears as a combination of two exponential distributions (two straight lines).

It is interesting to observe that the higher the values for inter-sampling instants $(\delta)$ the more exponential-like behavior observed as indicated by the straight line in the semi-log $y-$ axis. Clearly, the information of previous instants vanishes the more you wait for the next sampling instant, thus approaching memoryless behavior as shown in Fig. 9 bottom-right. Hence, when the sampling ratio is large enough, memorilessness may be assumed.

\section{Multi-user experiments}

Fig. 10 shows the CCDF of the transmission time duration of all ONUs when a different number of them is sensed. In this case, $\delta=0.1 E X(2.314 \mu \mathrm{s})$. Obviously, the higher amount of codes sensed under transmission, the more time it is necessary to wait until all of them have finished their transmission.

As shown, the CCDF remains the same during some time $(d \leq 20 \mu s$ ) when more than two users are sensed in the Bimodal packet size distribution (bottom-right).

\section{TOWARDS TRANSMISSION SCHEDULING}

As mentioned before, coherence of state can be used to schedule the transmission of the next packet, that is, to determine the most suitable packet release time in terms of bit error rate.

Essentially, when a number of $u$ users have been sensed active, the addition of a new user into the network substantially increases the BER due to interference (Fig. 5). Then, a given
ONU might decide to wait for a certain amount of time until one or more active users have finished transmitting their packets.

In this light, let $X_{1}, \ldots, X_{u}$ denote the random variables which represent the transmission duration of ONU number $1, \ldots, u$ respectively, and let $X_{1}<X_{2}<\ldots<X_{u}$ without loss of generality. Then, the $j$-th order statistic:

$$
f_{X_{j}}(t)=\frac{u !}{(j-1) !(u-j) !} f_{X}(t) F(t)^{j-1}(1-F(t))^{u-j}
$$

denotes the probability density function of the $j$-th packet to have finished its transmission. For the particular case of the exponential distribution, Eq. 20 yields:

$$
f_{X_{j}}(d)=\frac{u !}{(j-1) !(u-j) !} \mu e^{-\mu d}\left(1-e^{-\mu d}\right)^{j-1} e^{-\mu(u-j) d}
$$

Essentially, Eq. 21 gives the probability to have $j$ ONUs having finished its transmission, thus $u-j$ still under transmission. Hence, if for BER requirements, the system imposes a maximum of 17 ONUs under transmission at the same time (see Fig. 5), then Eq. 21 gives the amount of time required to wait such that $u-j$ ONUs or less are still under transmission.

As a numerical example, let $u=20$ denote the actual number of users active, as sensed by the 21-st user. Let user number 21 decide to schedule (delay) its packet to an instant such that the probability to have 11 users or less becomes $95 \%$. The order statistics defined above can be used to answer the following question: How long is it necessary to wait until only 11 users at most are present in the PON with $95 \%$ confidence?

Fig. 11 shows the order statistics $j \in\{1,5,9,13\}$ assuming that $u=20$ are observed active. For brevity, only exponential distributed packet sizes are considered in this example. As shown, at time $t=10 \mu \mathrm{s}$, the first five users are very likely to have completed its transmission with probability 1.0000 , $0.9978,0.9883,0.9568$ and 0.8844 respectively. However, the ninth and thirteenth users exhibit a probability of 0.2420 and 0.0063 to have completed the transmission of their packets. Hence, the ONU sensing the channel is $95 \%$ confident that, 

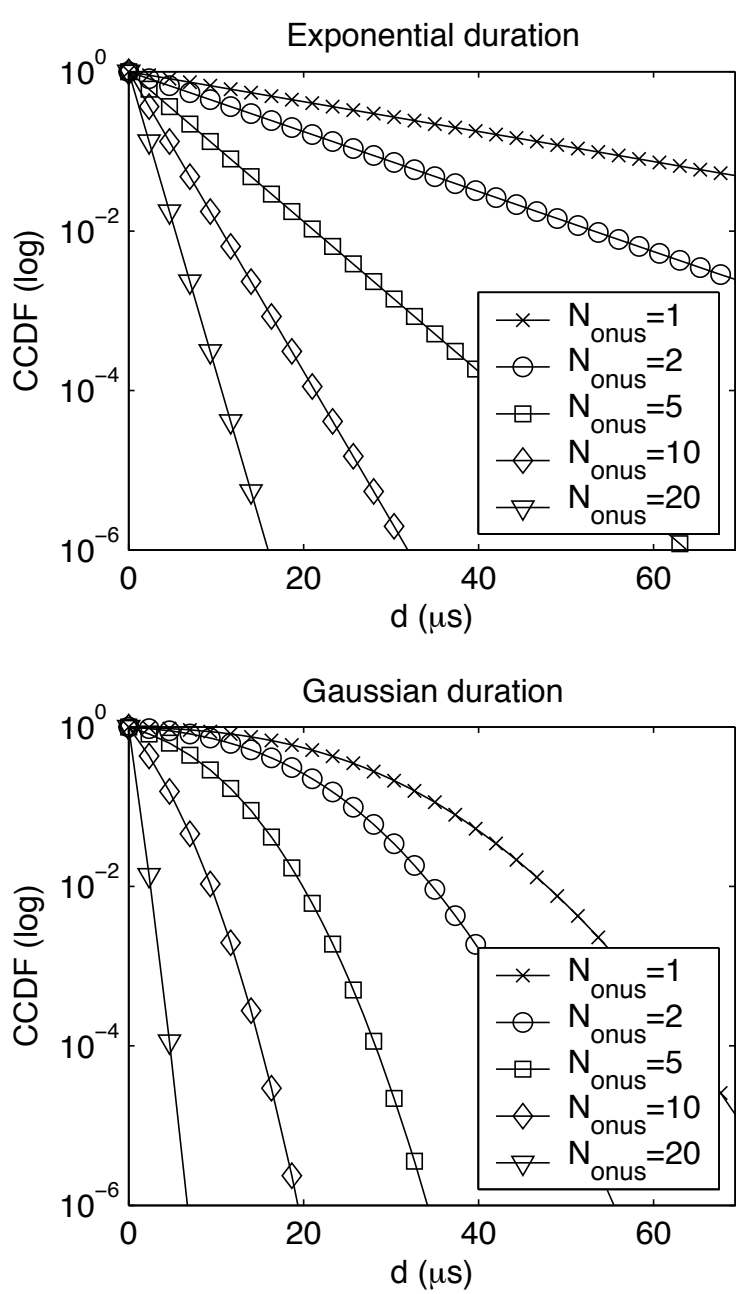
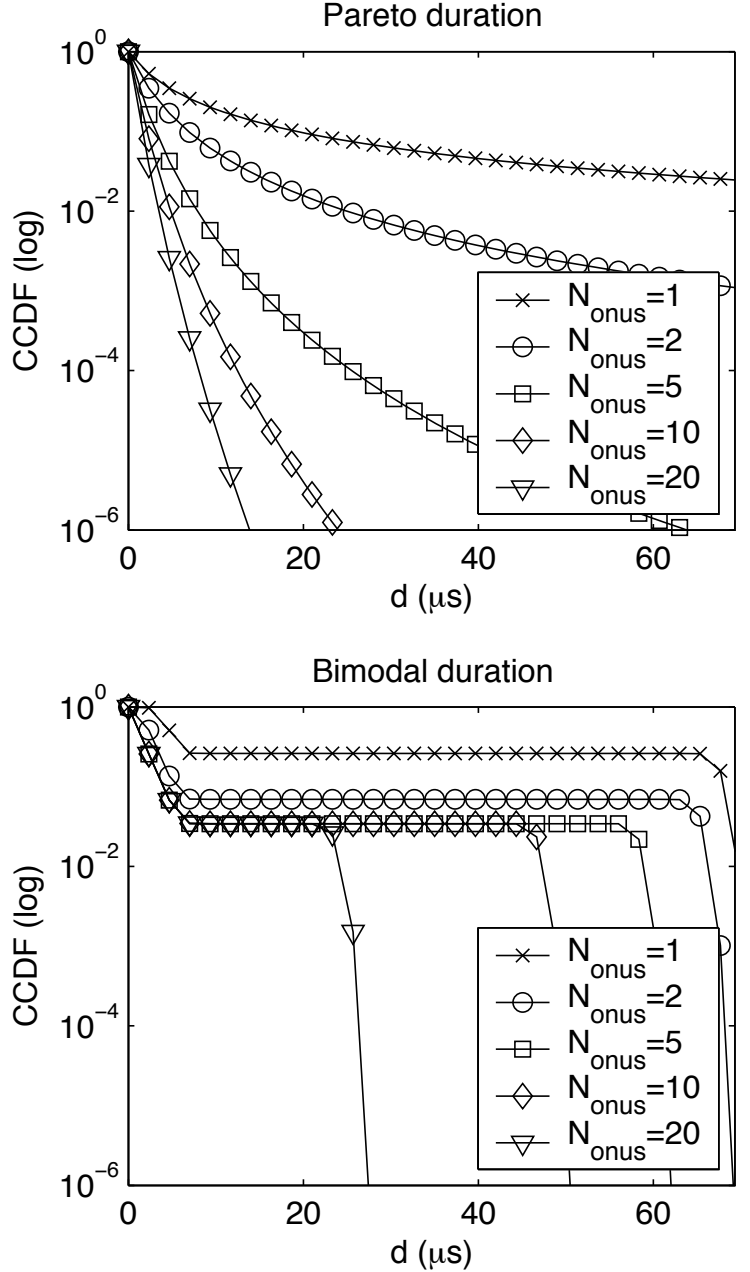

Fig. 10. Comparison when multiple ONUs are transmitting: Exponential (top-left), Pareto (top-right), Gaussian (bottom-left) and Bimodal (bottom-right).

at time $t=10 \mu \mathrm{s}$ (remember the propagation delay is approximately $5 \mu$ s if the ONUs are $1 \mathrm{~km}$ away from the optical merging point), about four ONUs have completed their transmission. As a result, there are no more than $20-4=16$ ONUs transmitting simultaneously in the PON under the assumption that no other ONU has started its transmission, of course. Thus, the ONU may decide to transmit at that time (16 users is still below the FEC limit see Fig. 5).

It is important to remark that such conclusions are only valid if no other ONU has started any transmission within the period of time of $10 \mu \mathrm{s}$. The reader should note that this probability is very small for such a short period of time.

\section{SUMMARY}

Optical technologies overcome the quality of service issues currently encountered in the access network. In this work, the future wired and wireless communication needs are reflected in personal, residential and business networks which all benefit from the deployment of an optical transparent access tier. We have presented, for the first time in full detail, an architecture based on PONs and spectral amplitude encoded optical codes. Additionally, transparent ONU-to-ONU communication is enabled in the sub-net of PONs with a single central office.
As shown throughout the work, the use of spectral codes gives raise to multiple user interference at the passive coupler of the PON. Essentially, the coherence between the observed state at the network node and the real (future) state at the merging point is evaluated at the packet level, thereby simplifying the analysis. A set of experiments are carried out for a number of realistic scenarios in case of four different packet size distributions and a fixed propagation delay between the network node and the merging point.

In all considered cases, the coherence of state is shown to depend on the packet size distribution and on the sampling rate. Regarding a single active user, the observed and future states are found to be coherent with respect to the transmission distance and memorylessness may be assumed for decreasing sampling rates. Concerning multiple active users, the coherence of state is shown to depend on the packet size distribution when the number of transmitting ONUs is small, but to approach exponential behaviour (memorylessness) as the number of simultaneous ONUs increases.

Additionally, it is shown that the a-priori knowledge of user activity can be exploited, for instance by allowing the ONUs to anticipate and decide whether or not to transmit a packet and, if so, when to schedule its transmission. Such scheduling must take into account the network design parameters, such as 

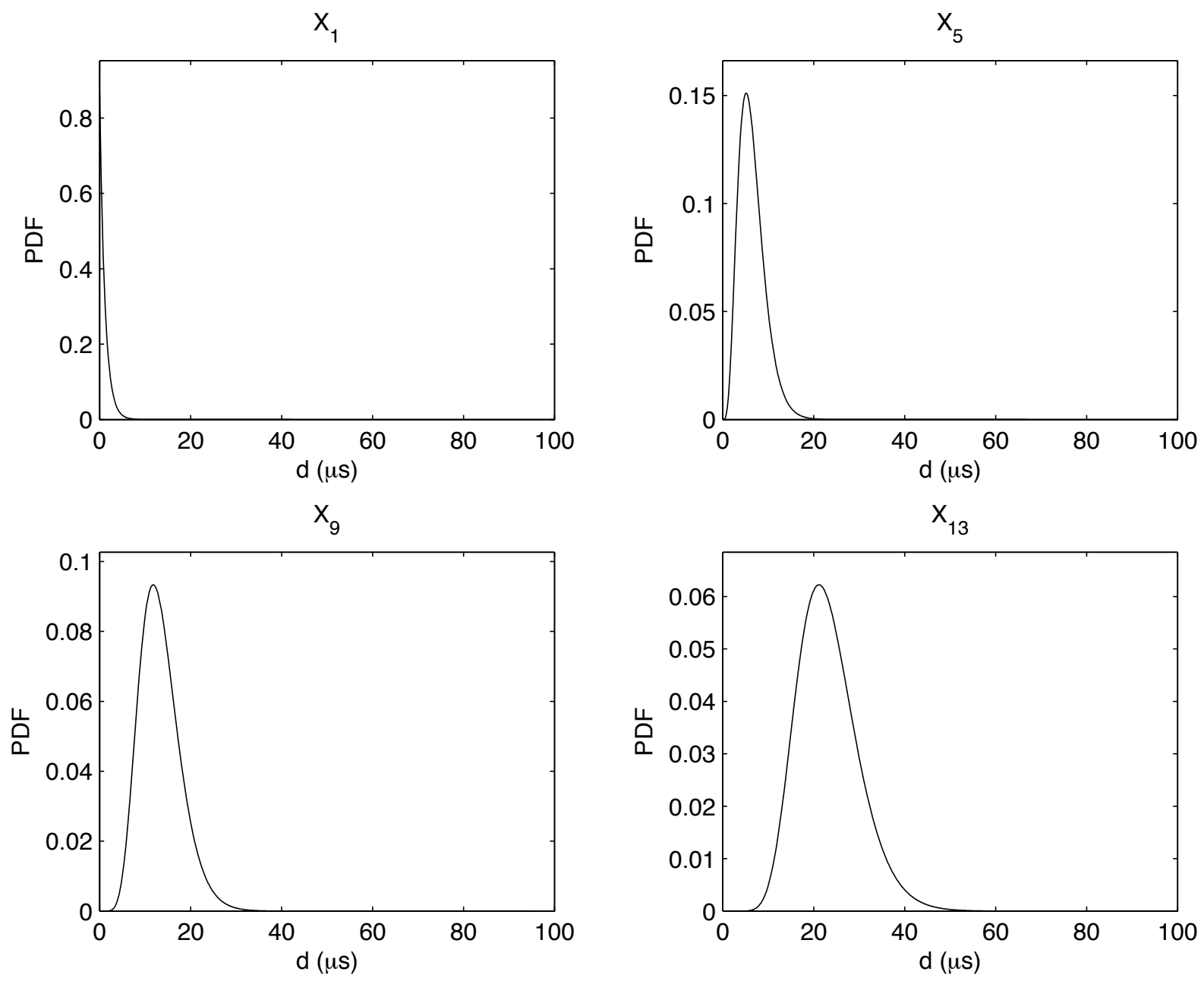

Fig. 11. Order statistics assuming $u=20 . f_{X_{1}}$ (top-left); $f_{X_{5}}$ (top-right); $f_{X_{9}}$ (bottom-left); and $f_{X_{13}}$ (bottom-right).

propagation delay and the number of ONUs per merging point. Finally, the mathematical framework introduced in this work sets the grounds for further development of medium access protocols in such transparent optical access architectures.

\section{ACKNOWLEDGEMENTS}

We gratefully acknowledge E. Tangdiongga and V. López Álvarez for valuable discussions.

\section{REFERENCES}

[1] R. E. Wagner, J. R. Igel, R. Whitman, M. D. Vaughn, A. B. Ruffin and S. Bickham, "Fiber-based broadband-access deployment in the United States," IEEE/OSA J. Lightw. Technol., vol. 24, no. 12, pp. 4526-4540, December 2006.

[2] M. Dischinger, A. Haeberlen, K. P. Gummadi, and S. Saroiu, "Characterizing residential broadband networks," in Proc. IMC 2007, October 2007, San Diego, CA, USA.

[3] J. M. Finochietto, J. Aracil, A. Ferreiro, J. P. Fernandez - Palacios Gimenez, and O. Gonzalez de Dios, "Migration strategies toward all optical metropolitan access rings," IEEE/OSA J. Lightw. Technol., vol. 25, no. 8, pp. 1918-1930, August 2007.

[4] M. J. O'Mahony, C. Politi, D. Klionidis, R. Nejabati and D. Simeonidou, "Future optical networks," IEEE/OSA J. Lightw. Technol., vol. 24, no. 12, pp. 4684-4696, December 2006.

[5] K. Cho, K. Fukuda, H. Esaki, and A. Kato, "The impact and implications of the growth in residential user-to-user traffic," in Proc. SIGCOMM 2006, September 2006, Pisa, Italy.
[6] Recommendation G.1010 End-user multimedia QoS categories, ITU-T, November 2001, Geneva, Switzerland.

[7] V. J. Hernandez, W. Cong, J. Hu, C. Yang, N. K. Fontaine, R. P. Scott, Z. Ding, B. H. Kolner, J. P. Heritage, S. J. B. Yoo, "A 320-Gb/s capacity (32-user $\times 10 \mathrm{~Gb} / \mathrm{s})$ SPECTS O-CDMA network testbed with enhanced spectral efficiency through forward error correction," IEEE/OSA J. of Lightw. Technol., vol. 25, no. 1, pp. 79-86, January 2007.

[8] N. Kataoka, N. Wada, X. Wang, G. Cincotti, A. Sakamoto, Y. Terada, T. Miyazaki and K.I. Kitayama, "Duplex, fully-asynchronous, 10Gbps $\times$ 8-user DPSK-OCDMA field trial using a multi-port en/decoder and SSFBG en/decoders," in Proc. OFC2008, post-deadline paper PDP27, March 2008, San Diego, CA, USA.

[9] P. Kamath, J. D. Touch, and J. A. Bannister, "State estimation in optical CDMA networks," ISI Technical Report ISI-TR-644, June 2007.

[10] G. Dán, V. Fodor, and G. Karlsson, "On the effects of the packet size distribution on FEC performance," Computer Networks, vol. 50, pp. 1104-1129, 2006.

[11] H. J. S. Dorren, Y. S. Oei, X. J. M. Leijtens, M. K. Smit, G. D. Khoe, Y. Liu, E. Tangdiongga, M. T. Hill, J. H. den Besten, J. H. C. van Zantvoort, E. Smalbrugge, T. de Vries, and J. J. M. Binsma, "All optical packet switching above $80 \mathrm{~Gb} / \mathrm{s}$," in Proc. ECOC 2006, paper Tu4.6.1 (invited), September 2006, Cannes, France.

[12] C. H. Lee, W. V. Sorin and B. Y. Kim, "Fiber to the home using a PON infrastructure," IEEE/OSA J. Lightw. Technol., vol. 24, no. 12, pp. 4568-4583, December 2006 (invited).

[13] A. M. J. Koonen, "Fiber to the home/fiber to the premises: what, where, and when?," Proc. IEEE, vol. 94, no. 5, pp. 911-934, May 2006 (invited).

[14] K. Fouli and M. Maier, "OCDMA and optical coding: principles, applications, and challenges," IEEE Commun. Mag., vol. 45, no. 8, pp. 27-34, August 2007. 
[15] Th. Zahariadis, "Evolution of the wireless PAN and LAN standards," Comp. Stand. and Interf., vol. 26, no. 3, pp. 175-185, May 2004.

[16] I. G. Niemegeers and S. M. Heemstra de Groot, "From personal area networks to personal networks: a user oriented approach," Wirel. Pers. Commun., vol. 22, pp. 175-186, August 2002.

[17] B. Huiszoon, F.T.H. den Hartog, M. García Larrodé, and A. M. J. Koonen, "Layer 2 and 3 contention resolution and radio-over-fiber in OCDMA PON for transparent optical access in personal networks," IEEE/OSA J. Lightw. Technol., vol. 26, no. 13, pp. 1752-1764, July 2008.

[18] G. Haßlinger, J. Mende, R. Geib, T. Beckhaus, F. Hartleb, Lecture notes in computer science, Springer Berling / Heidelberg, (Eds.) L. Mason, T. Drwiega, and J. Yan, pp. 998-1010: Measurement and characteristics of aggregated traffic in broadband access networks, September 2007.

[19] M. Jovanovic, F. Annexstein, and K. Berman, "Modeling peer-to-peer network topologies through small-world models and power laws," in $I X$ Telecommunications Forum TELFOR 2001, November 2001, Belgrade, Serbia.

[20] T. Karagiannis, P. Rodriguez, and K. Papagiannaki, "Should internet service providers fear peer-assisted content distribution?," in Proc. IMC 2005, October 2005, Berkely, CA, USA.

[21] Y. Liu, L. Xiao, L. M. Ni, and X. Zhang, "Location awareness in unstructured peer-to-peer systems," IEEE Trans. on Parall. and Distr. Syst., vol. 16, no. 2, pp. 163-174, February 2005.

[22] R. Cohen, "On the establishment of an access VPN in broadband access networks," IEEE Commun. Mag., vol. 41, no. 2, pp. 156-163, February 2003.

[23] Z. Zhang, Y. Q. Zhang, X. Chu, and B. Li, "An overview of virtual private network (VPN): IP VPN and optical VPN," J. Photon. Netw. Comm., vol. 7, no. 3, pp. 213-225, May 2004.

[24] T. Takeda, D. Brungard, D. Papadimitriou, and H. Ould-Brahim, "Layer 1 virtual private networks: driving forces and realization by GMPLS," IEEE Commun. Mag., vol. 43, no. 7, pp. 60-67, July 2005.

[25] N. Nadarajah, E. Wong, and A. Nirmalathas, "Implementation of multiple secure virtual private networks over passive optical networks using electronic CDMA," IEEE Photon. Technol. Lett., vol. 18, no. 3, pp. 484-486, February 2006.

[26] Y. Tian, X. Tian, L. Leng, T. Ye, and Y. Su, "Optical VPN connecting ONUs in different PONs," in Proc. OFC 2007, paper OWL6, March 2007, Anaheim, CA, USA.

[27] B. Huiszoon, H. de Waardt, G. D. Khoe and A. M. J. Koonen, "On the upgrade of an optical code division PON with a code-sense Ethernet MAC protocol," in Proc. OFC 2007, paper OWC7, March 2007, Anaheim, CA, USA

[28] B. Huiszoon, L. M. Augustin, E. A. J. M. Bente, H. de Waardt, G. D. Khoe, M. K. Smit and A. M. J. Koonen, "Integrated MachZehnder based spectral amplitude OCDMA on a passive optical network," IEEE J. of Sel. Topics in Quant. Electron., vol. 13, no. 5, pp. 1487-1496, September/October 2007.

[29] B. Huiszoon, Optically transparent multiple access networks employing incoherent spectral codes, Eindhoven University of Technology, June, 2008. Available online: Eindhoven University Library.

[30] P. Kamath, J. D. Touch, and J. A. Bannister, "The need for media access control in optical CDMA systems," in Proc. INFOCOM 2004, March 2004, Hong Kong, PR China.

[31] P. Saghari, P. Kamath, V. Arbab, M. Haghi, A. E. Willner, J. A. Bannister and J. D. Touch, "Experimental demonstration of an interferenceavoidance-based protocol for O-CDMA networks," in Proc. OFC 2006, paper PDP46, March 2006, Anaheim, CA, USA.

[32] C.F. Lam, Multi-wavelength optical code-division-multiple-access, Ph.D. thesis, UCLA, 1999.

[33] A. Tychopoulos, O. Koufopavlou, and I. Tomkos, "FEC in optical communications," IEEE Circuits and Devices Magazine, vol. 22, no. 6, pp. 79-86, November/December 2006.

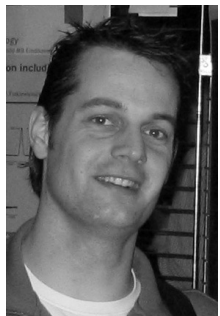

B. Huiszoon was born in Vlissingen, The Netherlands, in 1978. He received the M.Sc. and Ph.D. degrees from the department of Electrical Engineering, Eindhoven University of Technology, Eindhoven, The Netherlands, in 2003 and 2008. He has been a visiting researcher at the Networking Researching Group of the Universidad Autónoma de Madrid in Madrid, Spain, from September 2007 until June 2008 partly within the FP6 e-Photon/ONe+ Network of Excellence. His research interests are in the field of broadband optical access networks and systems, optical code division multiple access and ubiquitous broadband service delivery. He acts or acted as a reviewer for several international journals and major IEEE conferences. Besides his research activities, he has been a General Member in the first board of the IEEE/LEOS Benelux Student Chapter from November 2004 until May 2007. In July 2007 he was awarded the Valorisation Grant I by the Technology Foundation STW of the Dutch Ministry of Economic Affairs to support the valorization of innovative academic research. He received the prize for the best M.Sc. thesis of 2003 at the TU/e in April 2004. He is a 2008 IEEE/LEOS Graduate Student Fellow and a Student Member of the IEEE since 2004.

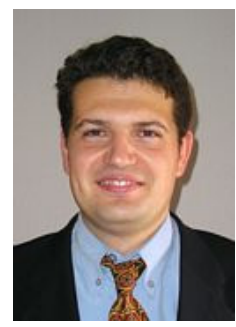

José Alberto Hernández completed the five-year degree in Telecommunications Engineering at Universidad Carlos III de Madrid (Spain) in 2002, and the Ph.D. degree in Computer Science at Loughborough University (Leics, United Kingdom) in 2005. After this, he joined the Networking Research Group at Universidad Autónoma de Madrid (Spain), where he actively participates in a number of both national and european research projects concerning the modeling and performance evaluation of communication networks, and particularly the optical burst switching technology. His research interests include the areas at which mathematical modelling and computer networks overlap.

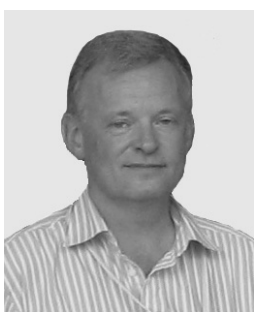

H. de Waardt was born in Voorburg, The Netherlands, in December, 1953. He received the M.Sc.E.E. and the Ph.D. degrees from the Delft University of Technology, The Netherlands in 1980 and 1995, respectively. In 1981, he started his professional carrier in the Physics Department at PTT Research in Leidschendam, The Netherlands, where he worked on the performance issues of opto-electronic devices. In 1989 he moved to the Transmission Department and became involved in WDM high-bit-rate optical transmission. In 1995 he was appointed as an Associated Professor at the Eindhoven University of Technology (TU/e), Eindhoven, The Netherlands in the area of highcapacity trunk transmission. He coordinated the participation of TU/e in ACTS Upgrade, ACTS BLISS, ACTS APEX and IST FASHION. Presently he serves as project leader of the national research initiative Freeband Broadband Photonics (2004-2008). His current interests are in high capacity optical transmission and networking, integrated optics and semiconductor optical amplifiers/modulators. He (co)authored over 150 conference and journal papers. Dr. de Waardt is member of the IEEE. 


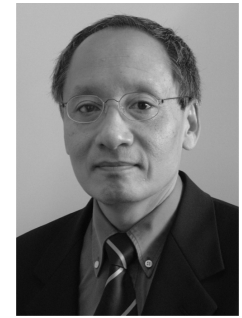

G.D. Khoe received the degree of Elektrotechnisch Ingenieur, cum laude, from the Eindhoven University of Technology, Eindhoven, The Netherlands, in 1971. He decided to start research at the Dutch Foundation for Fundamental Research on Matter (FOM) Laboratory on Plasma Physics, Rijnhuizen. In 1973 he moved to the Philips Research Laboratories to start research in the area of optical fiber communication systems. In 1983, he was appointed as part time professor at Eindhoven University of Technology. He became a full professor at the same University in 1994 and is currently chairman of the Department of Telecommunication Technology and Electromagnetics (TTE). Most of his work has been devoted to single-mode fiber systems and components. Currently his research programs are centered on ultrafast all-optical signal processing, high capacity transport systems and systems in the environment of the users. $\mathrm{He}$ has served in the IEEE/LEOS organization as European Representative in the BoG, VP Finance \& Administration, VP Membership, BoG Elected Member, President and member of the Executive Committee of the IEEE Benelux Section. He was founder of the LEOS Benelux Chapter. He has been an IEEE Fellow since 1991, OSA Fellow since 2006 and received the MOC/GRIN award in 1997.

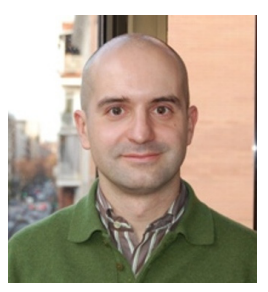

Javier Aracil received the M.Sc. and Ph.D. degrees (Honours) from Universidad Politécnica de Madrid, Spain in 1993 and 1995, both in Telecommunications Engineering. In 1995 he was awarded with a Fulbright scholarship and was appointed as a Postdoctoral Researcher of the Department of Electrical Engineering and Computer Sciences, University of California, Berkeley. In 1998 he was a research scholar at the Center for Advanced Telecommunications, Systems and Services of The University of Texas at Dallas. He has been an associate professor for University of Cantabria and Public University of Navarra and he is currently a full professor at Universidad Autónoma de Madrid, Madrid, Spain. His research interest are in optical networks and performance evaluation of communication networks. He has authored more than 75 papers in international conferences and journals.

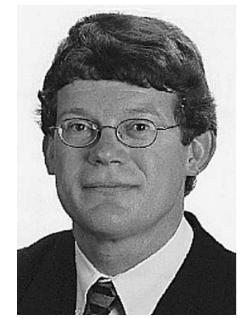

A.M.J. Koonen was born in Oss, The Netherlands, in 1954. He received the M.Sc. degree (cum laude) in electrical engineering from Eindhoven University of Technology, Eindhoven, The Netherlands, in 1979. In 1979, he joined Philips Telecommunicatie Industrie, part of which has become Lucent Technologies Network Systems, The Netherlands, since 1984. He worked on high-speed transmission systems and optical fiber systems for hybrid access networks. From 1987 to 2000, he was a Technical Manager in the Forward Looking Work Department, Bell Laboratories, Lucent Technologies Network Systems. From 1991 to September 2000, he was a Part-Time Professor at the University of Twente, Enschede, The Netherlands, holding a Chair in Photonic Networks. In September 2000, he joined the Eindhoven University of Technology as a Part-Time Professor and has been a Full-Time Professor since January 2001, holding a Chair in Broadband Communication Networks with the Telecommunication Technology and Electromagnetics Group, Department of Electrical Engineering. In 1999, he was appointed as Bell Laboratories Fellow. He has been an IEEE Member since September 2000, a Senior Member since May 2001 and a Fellow since January 2007. 